\title{
ANÁLISE DA DISSEMINAÇÃO DOS CONCEITOS DA INDÚSTRIA 4.0 ENTRE PROFISSIONAIS E ESTUDANTES DA ENGENHARIA DE PRODUÇÃO
}

\author{
João Barros de Melo Júnior (UFPE) jbmelojunior@ gmail.com \\ Brenda Luisa Alves Pinheiro de Melo (UFPE) brenda.luisa.melo@gmail.com \\ Gabriela Eloanne Vidal Leite (UFPE) gabrielaelo321@ gmail.com \\ Marcele Elisa Fontana (UFPE) marcele.elisa@ufpe.br
}

\section{Resumo}

As mudanças no mercado culminaram no aumento da competitividade entre as empresas, as quais para atender as necessidades dos clientes precisaram se adaptar às suas novas exigências. A fim de melhorar os processos e aumentar a produtividade, a Indústria 4.0 propõe o aumento do nível de eficiência da organização com o uso de algumas ferramentas e tecnologias como Internet das Coisas (IoT), Computação em Nuvem e Big Data que precisam ser dominadas pelos profissionais envolvidos no processo. A partir disso, o objetivo desse estudo foi avaliar a diferença no grau de disseminação dos conceitos da Indústria 4.0 entre estudantes e profissionais da engenharia de produção, possibilitando, assim, encontrar possíveis melhorias para que com o devido acesso às informações os profissionais e futuros profissionais possam se manter no mercado e contribuir com a adaptação às mudanças da indústria 4.0 para um sistema produtivo mais eficiente. Para tanto, realizou-se a análise estatística, buscando identificar a influência entre o perfil dos respondentes e variáveis de resposta. Como resultado percebeu-se uma maior disseminação dos conceitos da Indústria 4.0 entre profissionais, quando comparado aos estudantes universitários. O que sugere um possível ponto de melhoria, ampliando o acesso dos universitários aos conceitos da Indústria 4.0 .

Palavras-Chaves: Análise Estatística; Indústria 4.0; Internet das coisas; Manufatura avançada.

\section{Introdução}

A Indústria 4.0 impulsiona o crescimento e o desenvolvimento através da sua capacidade de eficiência. Diante disto, os sistemas de manufatura necessitam ser reconfigurados tornando-se capazes de absorver as informações e aplicá-las nos processos de fabricação, tornando os processos flexíveis, adaptáveis e inteligentes com a finalidade de atender o novo formato dinâmico e global de mercado consumidor. Esse sistema precisa oferecer um novo potencial à 
indústria manufatureira, como responder da melhor maneira os requisitos individuais dos clientes, aprimorar a tomada de decisões e acrescentar novas capacidades aos produtos (CONTRERAS et al. 2017).

Todavia, isso necessita que não somente os processos se adequem, mas também que os profissionais e futuros profissionais evoluam tecnicamente acompanhando a $4^{\mathrm{a}}$ Revolução Industrial. Isto é, se faz necessário que os envolvidos no processo estejam aptos a lidar com inovações como a Internet das Coisas (IoT), Computação em Nuvem, Big Data, etc. Em outra palavras, as novas habilidades exigidas pelo mercado de trabalho da indústria 4.0 requerem profissionais capazes de adaptar as transformações digitais inerentes a esta revolução e a academia necessita reavaliar e adequar suas ofertas formativas para estarem alinhadas às necessidades das futuras gerações de profissionais e do mercado de trabalho (RODRIGUES et al., 2017).

O objetivo geral deste trabalho foi avaliar a percepção dos profissionais e estudantes acerca das mudanças inerentes à Indústria 4.0, bem como vantagens e desafios enfrentados para sua implantação. Para isto, realizou-se uma fundamentação sobre o assunto. Em seguida foi elaborado um questionário aplicado de forma digital para identificar o grau de disseminação dos conceitos entre os profissionais e estudantes e, através de uma análise estatística analisar a relação entre o conhecimento dos conceitos e o fato de o participante estar inserido no mercado ou na universidade.

Este artigo está organizado da seguinte maneira: a secção 2 apresenta os temas abordados estão presentes na Fundamentação Teórica, a secção 3 apresenta o processo metodológico seguido durante a execução da pesquisa, a secção 4 apresenta os resultados obtidos e, por fim, a secção 5 apresenta as conclusões.

\section{Fundamentação teórica}

\subsection{Indústria 4.0}

Com a Indústria 4.0 a forma como os sistemas operam, como os produtos são fabricados e consumidos também mudarão. A mudança é tão drástica ao ponto de ter recebido o nome de Quarta Revolução Industrial (COELHO, 2016).

Devido à digitalização, $100 \%$ dos processos estarão conectados, desde o setor da produção até marketing e vendas. As máquinas conseguirão conversar entre si, estabelecer comunicação 
com peças, ferramentas e seres humanos. Isso será possível graças aos sistemas ciber-físicos que estabelecem a comunicação entre os dispositivos, enviando informações de um para o outro (DAVENPORT e KUDYBA, 2017).

Para o consumidor, o principal impacto está relacionado aos ganhos em flexibilidade, agilidade, eficiência produtiva e melhorias constantes de processo de distribuição com maior dinamismo à personalização dos produtos. As empresas poderão entregar algo personalizado na mesma velocidade que, hoje, produzem em série. Isso porque, novamente, as máquinas receberão diretamente os pedidos ou as informações de comportamento do cliente e oferecerão o que ele deseja (GILLIAND e WENZY, 2012).

Já os profissionais atuantes no mercado e os que estão ingressando no momento precisarão adaptar-se a esta nova realidade, visto que os principais impactos ocorrerão na força de trabalho qualificada, interferindo na empregabilidade e na necessidade de as pessoas aperfeiçoarem e desenvolverem suas habilidades e técnicas para operarem com todas as novas tecnologias e garantir sua empregabilidade (HECKLAU et al., 2016).

\subsection{Tecnologias de apoio à indústria 4.0}

$\mathrm{Na}$ indústria 4.0 existem tecnologias que a sustentam e tornam possível alcançar seus inúmeros benefícios. Dentre elas estão computação em nuvem, Big Data e internet das coisas.

Para Katzan (2010), a computação em nuvem significa que toda a infraestrutura e informação estarão disponíveis de forma digital na Internet, incluindo os softwares, ferramentas de busca, redes de comunicação, provedores, centros de armazenamento e processamento de dados, onde possui fácil acesso e os recursos podem ser dinamicamente reconfigurados e ajustados para uma escala variável de acordo com a sua utilização.

Tem como principal vantagem o custo visto que não necessita do planejamento e execução de projetos de infraestrutura interna de data centers nas organizações. Em contrapartida, segundo VAQUERO et al. (2009), as maiores desvantagens da computação em nuvem são pontos chave para a evolução e adoção da mesma, dentre elas estão: segurança, confiabilidade e disponibilidade.

Já o Big Data, através da coleta de dados com enorme velocidade de diversas fontes e formatos, contando com uma estrutura integrada que armazena, processa e gerencia todas as informações permite acessar os dados em tempo real para tomar decisões mais eficientes (LI, 2015). 
Além disso, melhora a capacidade de mineração de dados, coleta de grandes quantidades de informações de diferentes dispositivos, melhoria no processamento de dados e obtenção de informações (GALBRAITH, 2014).

Por fim, a Internet das coisas (IoT) transforma os objetos físicos em objetos inteligentes. Através dela e suas funcionalidades advindas de sensores e tecnologias de comunicação, qualquer segmento pode transformar o seu processo de negócios e fornecer controle sob suas operações, além da oportunidade de tomar decisões informadas e descentralizadas, com alto grau de qualidade (MIORANDI et al., 2012)

Tan e Wang (2010) identificaram importantes recursos essenciais para o funcionamento da IoT, como: confiabilidade, escalabilidade, modularidade, qualidade de serviço, integração e interoperabilidade, recursos de interface e rede, segurança. No entanto, eles acreditam que a segurança na IoT é uma questão crucial, pois é um recurso complementar e nenhuma solução técnica para segurança e privacidade das informações é garantida.

Os conceitos de Big Data, computação em nuvem e internet das coisas apresentados nesta secção são pilares da indústria 4.0 e, portanto, é importante conhecer o cenário no que diz respeito ao entendimento de profissionais e estudantes da engenharia de produção acerca destes conceitos, bem como seus fundamentos e sua importância para o sistema produtivo.

\section{Metodologia}

Nessa seção é apresentada a metodologia e as etapas da pesquisa, que nesse caso adotou-se o método de pesquisa de campo, análise descritiva e inferência estatística. A Figura 1 apresenta os passos necessários para conduzir o trabalho.

A pesquisa teve uma visão exploratória, em que segundo Beuren (2006, p. 80), "normalmente ocorre quando há pouco conhecimento sobre a temática a ser abordada”. Apoiou-se, também, na perspectiva descritiva, que segundo Beuren (2006), tem como principal objetivo a descrição das características de determinada população, estabelecendo relações entre as variáveis em questão. Além disso, esta pesquisa propõe-se, também, a analisar a influência do perfil dos entrevistados nas respostas.

Inicialmente realizou-se uma fundamentação teórica sobre Indústria 4.0 e os principais temas relacionados a fim de compreender a relação entre as tecnologias necessárias para a implementação da Manufatura Avançada. 
Figura 1 - Etapas da Pesquisa

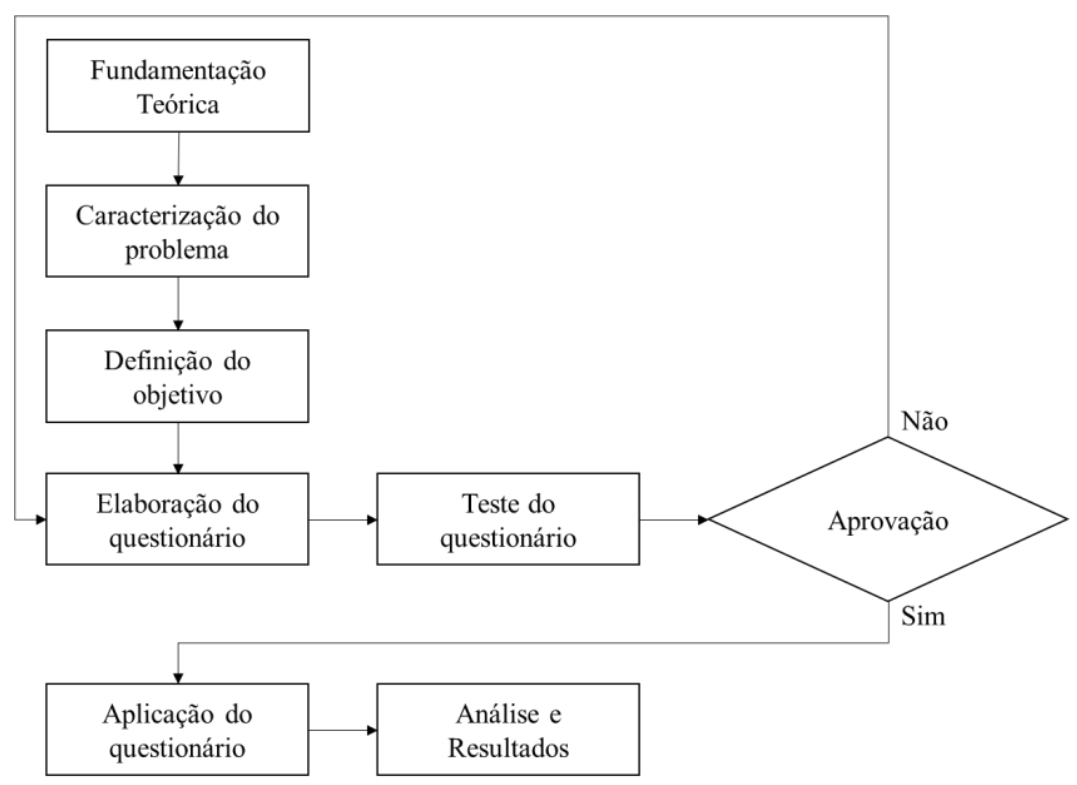

Fonte: Os Autores (2019)

O segundo passo foi caracterizar o problema, em que a partir das revoluções industriais e das várias tecnologias desenvolvidas os profissionais e estudantes precisam estar atentos às mudanças no mercado.

De acordo com a caracterização do problema, o objetivo foi definido como avaliar se há diferença no grau de disseminação dos conceitos da Indústria 4.0 entre estudantes e profissionais da engenharia de produção frente às mudanças provocadas pela quarta revolução industrial. Atualmente está em andamento a quarta revolução industrial, exigindo mais uma vez que os profissionais se adaptem às novas tecnologias.

Um questionário (Anexo I) foi, então, elaborado contendo dois blocos e baseado no estudo realizado pela Confederação Nacional da Indústria - CNI (2016), os quais foram:

- $\quad$ Bloco I - Dados do entrevistado- Idade, ano de formatura e ocupação atual;

- Bloco II - Conhecimento - Conhecimento de alguns conceitos da Indústria 4.0 bem como a avaliação de domínio das tecnologias.

Realizou-se um teste do questionário com 12 estudantes de Engenharia de Produção da Universidade Federal de Pernambuco, que explanaram suas dúvidas e indicaram a adequação do mesmo, para que o questionário pudesse, então, coletar melhor as informações pretendidas. E em seguida foi realizada a aplicação do questionário por meio de uma plataforma on-line, na plataforma Google Doc's, a uma amostra extraída por conveniência. Nele avaliaram-se as 
perguntas através da escala de Likert, em que as respostas partiram de (1) "discordo totalmente" até (5) "concordo totalmente".

Para responder ao questionário era necessário possuir vínculo, empregatício ou educacional, na cidade de Caruaru-PE, ser graduando (penúltimo ou último ano) ou graduado em Engenharia de Produção, e já ter tido contato com os conceitos da Indústria 4.0, analisado por meio de uma pergunta no questionário.

Foi obtido um total de 164 respondentes ao questionário, no período de 22 a 29 de novembro de 2019, em que os resultados obtidos foram tabulados e foi realizada uma análise utilizando teste Qui-quadrado de Pearson, que é um teste de hipóteses não paramétrico, para compreender como às inovações provocadas pela Indústria 4.0 são interpretadas pelos respondentes.

Utilizou-se a estatística inferencial para verificar a correlação entre as variáveis analisadas e o perfil dos respondentes por meio do teste de hipóteses não paramétrico. Os testes de hipóteses não paramétricos são conhecidos por testes livres de distribuição, permitem uma aplicação mais generalizada, pois são menos rígidos que os paramétricos (BARBETTA et al., 2010).

Realizou-se o teste de Shapiro-Wilk com auxílio do software SPSS Statistics, visando avaliar a normalidade dos dados e concluiu-se para todas as variáveis que os dados não seguem uma distribuição normal. Foi, então, possível analisá-las com o teste do Qui-quadrado de Pearson para descobrir se existe uma associação explicativa entre duas variáveis, utilizando nível de significância $(\alpha)$ de $5 \%$. Em que para um $p$-valor menor do que o nível de significância estabelecido se rejeita a hipótese nula.

Dada à necessidade de o teste Qui-quadrado possuir apenas frequências não nulas, as questões propostas no questionário foram reorganizadas em respostas de "concordância" e "não concordância". Foram consideradas como "não concordância" as respostas informadas como "discordo totalmente", "discordo parcialmente" e "indiferente", como "concordância" as informadas como "concordo parcialmente" e "concordo totalmente".

\section{Resultados e análises}

A partir dos critérios de exclusão estabelecidos para esta pesquisa foram utilizadas 119 de um total de 164 respostas. As respostas de cada pergunta do questionário foram organizadas em tabelas a fim de facilitar as análises futuras. 
A Tabela 1 representa os resultados para a pergunta com relação à caracterização dos respondentes, onde é possível visualizar que a maioria dos respondentes é jovem com menos de 30 anos. Tal fato é compreensível visto que o curso de graduação em engenharia de produção no país é relativamente recente, tendo apenas 10 anos no agreste de Pernambuco.

Tabela 1 - Classificação dos participantes por idade

\begin{tabular}{c|c|c|c|c|c|c}
\hline $\begin{array}{c}\text { Caracterização dos } \\
\text { participantes }\end{array}$ & Profissional & $\%$ & Estudante & $\%$ & Total & $\%$ \\
\hline Menos de 25 anos & 0 & $0 \%$ & 86 & $93 \%$ & 86 & $72 \%$ \\
\hline Entre 25 e 30 anos & 16 & $59 \%$ & 6 & $7 \%$ & 22 & $18 \%$ \\
\hline Entre 30 e 35 anos & 6 & $22 \%$ & 0 & $0 \%$ & 6 & $5 \%$ \\
\hline Acima de 35 anos & 5 & $19 \%$ & 0 & $0 \%$ & 5 & $4 \%$ \\
\hline Total & 27 & $100 \%$ & 92 & $100 \%$ & 119 & $100 \%$ \\
\hline
\end{tabular}

Fonte: Os Autores (2019)

Em seguida, analisaram-se as respostas à pergunta "a empresa\universidade que eu estou inserido possibilita que eu conheça, domine e aplique os conceitos" sobre Indústria 4.0, Computação em Nuvem, Internet das Coisas e Big Data.

A Tabela 2 apresenta os resultados para o conceito de Indústria 4.0, onde é possível perceber que a maioria dos participantes possui algum grau de discordância. $\mathrm{O}$ que pode ser explicado pela pesquisa de Camargo e Mendonça (2017) que observou que no Brasil pesquisadores de 65 instituições haviam produzido conteúdos relacionados a Indústria 4.0, enquanto que pesquisadores de apenas 2 instituições das cerca de 20 existentes no estado de Pernambuco produziram conteúdos relacionados ao tema, demonstrando a lenta evolução do acesso a estes conceitos na delimitação geográfica onde o questionário foi aplicado.

Tabela 2 - Domínio do conceito Indústria 4.0

\begin{tabular}{c|c|c|c|c|c|c}
\hline $\begin{array}{c}\text { Domínio do Conceito: } \\
\text { Indústria 4.0 }\end{array}$ & Profissional & $\%$ & Estudante & $\%$ & Total & $\%$ \\
\hline Discordo totalmente & 2 & $7 \%$ & 4 & $4 \%$ & 6 & $5 \%$ \\
\hline Dis cordo parcialmente & 7 & $26 \%$ & 19 & $21 \%$ & 26 & $22 \%$ \\
\hline Indiferente & 8 & $30 \%$ & 27 & $29 \%$ & 35 & $29 \%$ \\
\hline Concordo parcialmente & 5 & $19 \%$ & 23 & $25 \%$ & 28 & $24 \%$ \\
\hline Concordo totalmente & 5 & $19 \%$ & 19 & $21 \%$ & 24 & $20 \%$ \\
\hline Total & 27 & $100 \%$ & 92 & $100 \%$ & 119 & $100 \%$ \\
\hline
\end{tabular}

Fonte: Os Autores (2019)

Com isso, a Hipóteses A pode ser formulada como sendo: 
- $\quad \mathrm{HA}_{0}: \mathrm{O}$ respondente ser profissional ou estudante não influencia no domínio do conceito Indústria 4.0;

- $\quad \mathrm{HA}_{1}$ : O respondente ser profissional ou estudante influencia no domínio do conceito Indústria 4.0.

A Tabela 3 apresenta a relação entre o perfil dos respondentes e sua opinião sobre o seu domínio do conceito de Indústria 4.0

Tabela 3 - Perfil vs Domínio do conceito Indústria 4.0

\begin{tabular}{|c|c|c|c|c|}
\hline & \multicolumn{2}{|c|}{ Indústria 4.0} & \multirow[b]{2}{*}{ Total } \\
\hline & & Não concordo & Concordo & \\
\hline \multirow{2}{*}{ Perfil } & Estudante & 50 & 42 & 92 \\
\hline & Profissional & 17 & 10 & 27 \\
\hline & Total & 67 & 52 & 119 \\
\hline
\end{tabular}

Obteve-se o valor $p=0,427$ e $\alpha=0,005$ como valor $p>\alpha$ deve-se rejeitar HA $\mathrm{H}_{1}$ Logo, a hipótese de que o perfil do respondente influencia o domínio do conceito Indústria 4.0 deve ser rejeitada.

Em seguida, a Tabela 4 mostra os resultados para o conceito de Computação em Nuvem.

Tabela 4 - Domínio do conceito Computação em Nuvem

\begin{tabular}{c|c|c|c|c|c|c}
\hline $\begin{array}{c}\text { Domín io do Conceito: } \\
\text { Computação em nuvem }\end{array}$ & Profis sional & $\%$ & Estudante & $\%$ & Total & $\%$ \\
\hline Dis cordo totalmente & 4 & $15 \%$ & 24 & $26 \%$ & 28 & $24 \%$ \\
\hline Discordo parcialmente & 5 & $19 \%$ & 21 & $23 \%$ & 26 & $22 \%$ \\
\hline Indiferente & 4 & $15 \%$ & 25 & $27 \%$ & 29 & $24 \%$ \\
\hline Concordo parcialmente & 8 & $30 \%$ & 17 & $18 \%$ & 25 & $21 \%$ \\
\hline Concordo totalmente & 6 & $22 \%$ & 5 & $5 \%$ & 11 & $9 \%$ \\
\hline Total & 27 & $100 \%$ & 92 & $100 \%$ & 119 & $100 \%$ \\
\hline
\end{tabular}

Fonte: Os Autores (2019)

Com isso, a Hipóteses B pode ser formulada como sendo:

- $\quad \mathrm{HB}_{0}: \mathrm{O}$ respondente ser profissional ou estudante não influencia no domínio do conceito Computação em nuvem;

- $\quad \mathrm{HB}_{1}$ : O respondente ser profissional ou estudante influencia no domínio do conceito Computação em nuvem.

A Tabela 5 apresenta a relação entre o perfil dos respondentes e sua opinião sobre o seu domínio do conceito de Computação em Nuvem. 
Tabela 5 - Perfil vs Domínio do conceito Computação em

\begin{tabular}{cc|c|c|c}
\multirow{2}{*}{} & \multicolumn{2}{|c|}{ Computação em nuvem } & \multirow{2}{*}{} \\
\cline { 3 - 5 } & \multicolumn{2}{c}{ Não concordo } & Concordo & Total \\
\hline \multirow{2}{*}{ Perfil } & Estudante & 70 & 22 & 92 \\
\cline { 2 - 5 } & Profissional & 13 & 14 & 27 \\
\hline \multirow{2}{*}{ nuvem } & Total & 83 & 36 & 119
\end{tabular}

Fonte: Os Autores (2019)

Obteve-se valor $p=0,005$. Com o nível de significância 5\%, como valor $p<\alpha$, rejeita-se $\mathrm{HB}_{0}$. Assim, não se deve rejeitar a hipótese que o perfil do respondente influencia o domínio do conceito em questão.

Na Tabela 6 mostra o conceito de Internet das Coisas.

Tabela 6 - Domínio do conceito Internet das Coisas

\begin{tabular}{c|c|c|c|c|c|c}
\hline $\begin{array}{c}\text { Domínio do Conceito: } \\
\text { Intemet das coisas }\end{array}$ & Profissional & $\%$ & Estudante & $\%$ & Total & $\%$ \\
\hline Discordo totalmente & 3 & $11 \%$ & 10 & $11 \%$ & 13 & $11 \%$ \\
\hline Dis cordo parcialmente & 7 & $26 \%$ & 17 & $18 \%$ & 24 & $20 \%$ \\
\hline Indiferente & 3 & $11 \%$ & 25 & $27 \%$ & 28 & $24 \%$ \\
\hline Concordo parcialmente & 9 & $33 \%$ & 27 & $29 \%$ & 36 & $30 \%$ \\
\hline Concordo totalmente & 5 & $19 \%$ & 13 & $14 \%$ & 18 & $15 \%$ \\
\hline Total & 27 & $100 \%$ & 92 & $100 \%$ & 119 & $100 \%$ \\
\hline
\end{tabular}

Fonte: Os Autores (2019)

Com isso, a Hipóteses $\mathrm{C}$ pode ser formulada como sendo:

- $\quad \mathrm{HC}_{0}: \mathrm{O}$ respondente ser profissional ou estudante não influencia no domínio do conceito Internet das coisas;

- $\quad \mathrm{HC}_{1}$ : $\mathrm{O}$ respondente ser profissional ou estudante influencia no domínio do conceito Internet das coisas.

A Tabela 7 apresenta a relação entre o perfil dos respondentes e sua opinião sobre o seu domínio do conceito de Internet das Coisas.

Tabela 7 - Perfil vs Domínio do conceito Internet das coisas

\begin{tabular}{cc|c|c|c}
\multirow{2}{*}{} & \multicolumn{2}{|c|}{ Internet das coisas } & \multirow{2}{*}{} \\
\cline { 3 - 5 } & Não concordo & Concordo & Total \\
\hline \multirow{2}{*}{ Perfil } & Estudante & 52 & 40 & 92 \\
\cline { 2 - 5 } & Profissional & 13 & 14 & 27 \\
\hline \multirow{2}{*}{ Total } & 65 & 54 & 119
\end{tabular}

Fonte: Os Autores (2019) 
Para valor $p=0,442$, com o nível de significância 5\%. Como valor $p>\alpha$ rejeita-se $\mathrm{HC}_{1}$. Logo, deve-se rejeitar hipótese que o perfil do respondente influencia o domínio do conceito em questão.

Por fim, a Tabela 8 apresenta os resultados para o conceito de Big Data.

Tabela 8 - Domínio do conceito Big Data

\begin{tabular}{c|c|c|c|c|c|c}
\hline $\begin{array}{c}\text { Domín io do Conceito: } \\
\text { Big Data }\end{array}$ & Profissional & $\%$ & Estudante & $\%$ & Total & $\%$ \\
\hline Discordo totalmente & 5 & $19 \%$ & 14 & $15 \%$ & 19 & $16 \%$ \\
\hline Dis cordo parcialmente & 4 & $15 \%$ & 16 & $17 \%$ & 20 & $17 \%$ \\
\hline Indiferente & 3 & $11 \%$ & 18 & $20 \%$ & 21 & $18 \%$ \\
\hline Concordo parcialmente & 10 & $37 \%$ & 27 & $29 \%$ & 37 & $31 \%$ \\
\hline Concordo totalmente & 5 & $19 \%$ & 17 & $18 \%$ & 22 & $18 \%$ \\
\hline Total & 27 & $100 \%$ & 92 & $100 \%$ & 119 & $100 \%$ \\
\hline
\end{tabular}

Fonte: Os Autores (2019)

Com isso, a Hipóteses D pode ser formulada como sendo:

- $\quad \mathrm{HD}_{0}$ : O respondente ser profissional ou estudante não influencia no domínio do conceito Big Data;

- $\quad \mathrm{HD}_{1}$ : $\mathrm{O}$ respondente ser profissional ou estudante influencia no domínio do conceito Big Data.

A Tabela 9 apresenta a relação entre o perfil dos respondentes e sua opinião sobre o seu domínio do conceito de Big Data.

Tabela 9 - Perfil vs Domínio do conceito Big Data

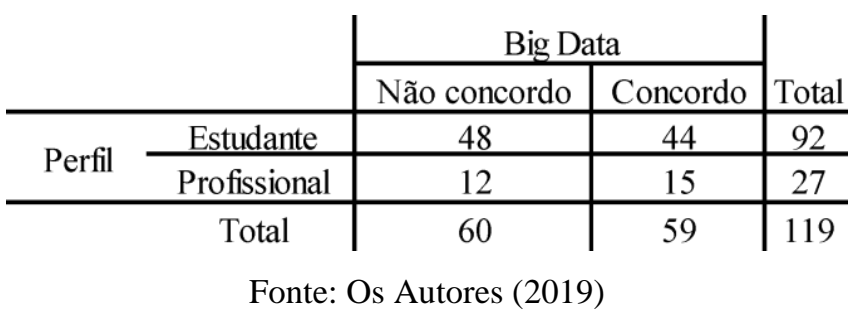

Obteve-se o valor $p=0,480$. Com $\alpha$ de 5\%, como valor $p>\alpha$, rejeita-se $\mathrm{HD}_{1}$. Assim, deve-se rejeitar hipótese que o perfil do respondente influencia o domínio do conceito em questão.

De acordo com as análises realizadas foi percebido que há diferença na percepção dos conceitos relacionados à indústria 4.0 entre profissionais e estudantes. Pôde-se também inferir que a hipótese de que o perfil do respondente influencia no entendimento dos conceitos não foi rejeitada nas avaliações dos conceitos indústria 4.0, internet das coisas e Big Data. Além 
disso, a hipótese de que o perfil do respondente não influencia no entendimento dos conceitos foi rejeitada quando avaliado o conceito de computação em nuvem, tendo em vista que não é uma tecnologia recente, tendo sido mencionada pela primeira no ano de 1997 e, atualmente, vem crescendo cada vez mais como uma solução quase inquestionável para a utilização de recursos e capacidades das organizações.

\section{Conclusões}

A Indústria 4.0 possibilita o surgimento de novidades que influenciam o modo de produzir em todos os segmentos. Sendo assim, avaliar e entender como essas influências impactam o sistema produtivo, o mercado e, consequentemente, a forma como as instituições de ensino veem essa nova revolução, bem como, os profissionais e futuros profissionais da área é de suma importância. Por isso, o presente trabalho buscou avaliar a percepção dos profissionais e estudantes acerca dos conceitos inerentes à Indústria 4.0.

As tecnologias da indústria 4.0 apresentam soluções para alguns dos desafios que a sociedade lida atualmente em áreas como saúde, mobilidade urbana e eficiência enérgica com a implantação de redes elétricas inteligentes. Entretanto, os impactos sociais ocorrerão principalmente na força de trabalho, empregabilidade e necessidade das pessoas buscarem aperfeiçoamento nas suas competências para utilizar as novas tecnologias e assegurar sua empregabilidade, bem como o oferecimento de produtos e serviços cada vez mais eficientes.

Ao avaliar se os respondentes possuem conhecimento sobre os conceitos da Indústria 4.0 pôde-se concluir que há maior disseminação no mercado, visto que, em todos os conceitos pesquisados menos de $50 \%$ dos estudantes concordou dominar os conteúdos parcialmente ou totalmente.

Pode-se inferir através das análises estatísticas, em que a hipótese inicial de que o perfil influencia na percepção dos conceitos acerca da indústria 4.0 não foi rejeitada ao avaliar o conceito de computação em nuvem, mas foi rejeitada quando avaliada nos conceitos de indústria 4.0, internet das coisas e Big Data. Conclui-se, então, que embora exista diferença na disseminação dos conceitos analisados, estatisticamente não se pode afirmar a existência de uma relação entre ser profissional ou estudante e o conhecimento acerca de conceitos inseridos no contexto da indústria 4.0. Além disso, devido ao tamanho e representatividade da amostra, não se pode fazer afirmações sobre a população, mas apenas levantar indícios. 
Através dos resultados obtidos é possível, então, conhecer o cenário atual quanto à disseminação dos conceitos avaliados e, assim, identificar pontos de melhoria para as universidades no acesso a estes conceitos, como também os profissionais que devem estar na constante busca por novas informações tornando mais eficiente à transição para a manufatura avançada futuramente.

\section{REFERÊNCIAS}

BARBETTA, Pedro A.; REIS, Marcelo M.; BORNIA, Antônio C. Estatística para cursos de Engenharia de Informática. 3. Ed. São Paulo: Atlas, 2010.

BEUREN; Ilse M. Como elaborar trabalhos monográficos em contabilidade: teoria e prática. São Paulo: Atlas, 2006. 3. ed.

CAMARGO, Marcelo; MENDONÇA, Samara. Mapping of the research institutions that develop the theme Industry 4.0 in Brazil. 2017. Trabalho de Conclusão de Curso (Graduação em Administração.) - Faculdade de Ciências Aplicadas. Universidade Estadual de Campinas. Limeira, 2017.

COELHO, Pedro Nogueira M. Rumo à Indústria 4.0. Dissertação apresentada para a obtenção do grau de Mestre em Engenharia e gestão Industrial pela Universidade de Coimbra. Coimbra, p. 1-65. 2016.

CONTRERAS, J. D.; GARCIA, J. I.; PASTRANA, J.D. Developing of Industry 4.0 Applications, International Journal of Online Engineering - v. 13, n. 10, 2017.

CNI - CONFEDERAÇÃO NACIONAL DA INDÚSTRIA. (2016). Pesquisa inédita da CNI mostra cenário da indústria 4.0 no Brasil. Agência de Notícias CNI. Disponível em:

$<$ https://noticias.portaldaindustria.com.br/noticias/inovacao-e-tecnologia/pesquisa-inedita-da-cni-mostra-cenarioda-industria-40-no-brasil/> acesso em 31/08/2019.

DAVENPORT, Thomas H.; KUDYBA, Stephan. Designing and Developing Analytics-Based Data Products. MitMitsloan Management Review, Massachustes, v. Special Collection, p. 6-11, Julho 2017.

GALBRAITH, Jay R., Organizational Design Challenges Resulting from Big. Journal of Organization Design, Vol. 3, No. 1 (2014), pp. 2-13.

GILLIAND, Guy; WENZY, Holger. Transformation the business model for IT services. Boston Consulting Group. Boston, p. 1-4. 2012.

HECKLAU, Fabian; GALEITZKE, Mila; FLACHS, Sebastian; KOHL, Holger. Holistic approach for human resource management in Industry 4.0. Procedia CIRP, v. 54, p. 1-6, 2016.

KATZAN J.R, Harry. On an ontological view of cloud computing. Journal of Service Science[S.I.], v. 3, n. 1, p. 1-6, 2010.

LI, Jingran, Tao, Fei, Cheng, Ying, \& Zhao, Lianglin. Big Data in product lifecycle management. International Journal of Advanced Manufacturing Technology, v. 81, p. 1-4, p. 667-684, 2015.

MIORANDI, Daniele; SICARI, Sabrina; DE PELLEGRINI, Francesco; CHLAMTAC, Imrich. Internet of Things: vision, applications and research challenges, Ad Hoc Networks, Vol. 10 n. 7, 2012. pp. 1497-1516.

RODRIGUES, Gonçalo; CARVALHO, Bruno; REIGOTO, Andreia; ELIAS, Ana; BATISTA, Pedro; JARDIM, Sandra; MADEIRA, Nuno. Formação no Instituto Politécnico de Tomar: Alinhamento de competências para 
responder aos desafios da Indústria 4.0. Superavit: revista de gestão e ideias, Tomar, v. 2, n. 2, p.65-75, out. 2017.

TAN, Lu; WANG, Neng. Future internet: the Internet of Things, 3rd International Conference on Advanced Computer Theory and Engineering (ICACTE), pp. V5-376-V5-380. 2010

VAQUERO, Luis M.; RODERO-MERINO, Luis; CACERES, Juan; LINDNER, Maik. A Break in the Clouds: Towards a Cloud Definition. ACM SIGCOMM Computer Communication Review, 39(1): 50-55, Janeiro 2009. 


\section{ANEXO A - Questionário - Avaliação da percepção dos profissionais e estudantes acerca da indústria 4.0. \\ BLOCO I - Dados do entrevistado}

1. Qual o curso de sua formação?

2. Qual a sua idade?

3. Qual o ano/previsão de sua formação?

4. Qual a sua instituição de ensino/empresa de atuação?

5. Qual a sua cidade de atuação?

6. Como você se classifica no âmbito da engenharia de produção?

( ) Estudante universitário

( ) Profissional

\section{BLOCO II - Conhecimento}

1. Você já ouviu falar na indústria 4.0 ou na quarta revolução industrial ou manufatura avançada?
( ) SIM
( ) NÃO

2. A empresaluniversidade que eu estou inserido possibilita que eu conheça, domine e aplique os conceitos (avalie de 1 - discordo totalmente a 5 - concordo totalmente):

Indústria 4.0

Cloud Computing

Internet das Coisas

Big Data
( ) 1 ( ) 2 ( ) 3 ( ) 4 ( ) 5

( ) 1 ( ) 2 ( ) 3 ( ) 4 ( ) 5

( ) 1 ( ) 2 ( ) 3 ( ) 4 ( ) 5

( ) 1 ( ) 2 ( ) 3 ( ) 4 ( ) 5 